\title{
Appendiceal Goblet Cell Carcinoid Tumour: A Case of Unexpected Lung Metastasis
}

\author{
Marinos Pericleous ${ }^{a}$ Heather Lumgair ${ }^{a}$ Alex Baneke ${ }^{a}$ \\ Luke Morgan-Rowe $^{b}$ Martyn E. Caplin ${ }^{a}$ Tu Vinh Luong ${ }^{c}$ \\ Christina Thirlwell $^{d}$ Roopinder Gillmore ${ }^{d}$ \\ Christos Toumpanakis ${ }^{a}$ \\ ${ }^{a}$ Department of Neuroendocrine Tumours, European Center of Excellence, and \\ Departments of ${ }^{b}$ Radiology, ${ }^{c}$ Pathology and ${ }^{d}$ Oncology, Royal Free Hospital, \\ London, UK
}

\section{Key Words}

Appendiceal neuroendocrine tumours · Goblet cell · Carcinoid tumour

\begin{abstract}
Goblet cell carcinoid tumours are often considered a subset of appendiceal neuroendocrine tumours which behave more aggressively. They usually metastasize through transcoelomic/peritoneal invasion and common sites include the ovaries, peritoneum, and liver. Metastases may have goblet cell carcinoid, signet ring cell carcinoma or classic carcinoid histology. We report the first case in the literature of a patient with a goblet cell carcinoid with lung metastasis, which was associated with unfavourable outcome.
\end{abstract}

\section{Introduction}

Appendiceal neuroendocrine tumours (NETs) are the commonest tumours of the appendix and may be found in $0.3-0.9 \%$ of all appendicectomy samples [1]. About $6 \%$ of these share mixed histologic features of bot mucinous glandular and neuroendocrine differentiation and are commonly termed as appendiceal goblet cell carcinoids (GCCs) [2]. Other names have been used in the literature for this type of tumour, such as adenocarcinoid, mucinous carcinoid, crypt cell carcinoma, and mucin-producing NET. The tumour is thought to arise from pluripotent intestinal epithelial crypt-base stem cells. The term 'goblet cell carcinoid' was first coined in 1974 by Subbuswamy et al. [3] and is still accepted by the current WHO classification.

Marinos Pericleous, BSc, MBBS, MRCP(UK)
Department of Neuroendocrine Tumours, European Center of Excellence Royal Free Hospital

Pond Street, London NW3 2QG (UK)

Tel. +44 772590 0973, E-Mail pericleousmarinos@gmail.com 
The median age of presentation is in the 5th decade with a second peak in the 7th decade without any sex predilection [4]. Patients with GCCs are usually diagnosed incidentally following a surgical presentation (e.g. appendicitis, bowel obstruction/perforation or abdominal pain) $[5,6]$.

There is only one published report of lung metastasis to date, but it was retrospectively considered as an adenocarcinoma rather than a GCC. It was previously considered unusual to have lung metastases; however, the general literature on GCC is small. Here, we present the first histologically proven lung metastasis from a 'typical' GCC.

\section{Case Description}

A 44-year-old woman with a long-standing history of lower abdominal pain was initially referred to the gynaecology department for an ultrasonography of the abdomen/pelvis arranged by her local practitioner. The scan demonstrated bilateral ovarian cysts with features suspicious for malignancy. Levels of serum CA 125 and CA 19-9 were raised at that time and the patient underwent a bilateral oophorectomy and salpingectomy. Histology showed both ovaries massively infiltrated by mucinpositive tumour nests admixed with scattered synaptophysin-/chromogranin-positive neuroendocrine cells. The proliferation fraction with MIB1 immunostain was $<5 \%$. These features were interpreted as metastasis from a primary appendiceal GCC.

On the basis of these findings, the patient was referred to our neuroendocrine tumour unit where the possibility of further surgery (appendicectomy and right hemicolectomy) was discussed. At the time of referral, the patient had fully recovered from her recent surgery and was asymptomatic. In order to assess the disease status, we arranged for the patient to have a triple phase contrast CT of the chest, abdomen, and pelvis as well as functional imaging including indium-111 pentetreotide scinitigraphy (Octreoscan ${ }^{\mathrm{TM}}$ ) and ${ }^{18} \mathrm{FDG}$-PET. The CT scan demonstrated a malignant-looking right lung hilar lesion which measured $4.0 \times 1.8 \mathrm{~cm}$ ( $\underline{\text { fig. } 1)}$ ) and also multiple intra-abdominal lymph nodes measuring up to $1 \mathrm{~cm}$. Octreoscan was negative. ${ }^{18 F D G-P E T ~ d e m o n s t r a t e d ~ l o w-t o-m o d e r a t e ~ a v i d i t y ~ i n ~}$ the noted hilar lesion and there was no FDG uptake elsewhere (fig. 2). Serum chromogranin A was normal, whilst the serum levels of CA 19-9 and CA 125 were elevated at 4,405 IU/ml (normal: 0-37 $\mathrm{IU} / \mathrm{ml}$ ) and $205 \mathrm{IU} / \mathrm{ml}$ (normal: $<30 \mathrm{IU} / \mathrm{ml}$ ), respectively.

The possibility of a separate lung pathology was considered and the lung lesion was biopsied bronchoscopically. Histology showed metastatic GCC (fig. 3 ) and similar features to those of the ovarian metastases.

Following discussion in the multidisciplinary team meeting, a decision not to undergo further surgical intervention was taken and the patient was referred to the oncology team for systemic chemotherapy. A pretherapy contrast CT showed interlobular interstitial/septal thickening within the right lung (fig. 1) as well as bilateral pleural and pericardial effusions and ascites.

Systemic chemotherapy was initiated with a combination of folinic acid, 5-fluorouracil, and oxaliplatin (FOLFOX). The patient received 11 cycles of palliative FOLFOX in total, which was eventually stopped due to symptomatic deterioration. Further CT imaging at that time showed an unchanged lung lesion; however, the patient had new bony lesions, widespread peritoneal disease, pleural effusions, and ascites. A second-line chemotherapy regimen was considered but not given due to the patient's poor performance status. The patient underwent symptomatic/palliative management of the pleural effusions and ascites. She was discharged at the local hospice where she died 11 months after the initial diagnosis. 


\section{Discussion}

Appendiceal GCCs usually behave more aggressively than conventional appendiceal NETs. According to a suggested classification and on the basis of tumour cell differentiation and Ki67, GCCs have been divided into 'typical GCC' and 'adenocarcinomas ex-GCC' of either 'signet ring cell type' or 'poorly differentiated adenocarcinoma type' [7]. The two latter types have been associated with the worst prognosis.

As soon as the histopathological diagnosis is confirmed, according to recent guidelines [8], patients with GCCs are usually offered further prophylactic surgery usually right hemicolectomy and/or bilateral salpingo-oophorectomy. In contrast to the common appendiceal carcinoids, GCC run an aggressive course and quite often metastasize. The ovaries [9] and peritoneum represent the most common metastatic sites. GCC metastases have also been reported in the liver [10], ribs, vertebrae, and prostate, whilst in one case GCC cells were found in the pleural fluid as well [11].

Debulking surgery with adjuvant intraperitoneal chemotherapy may improve survival in some patients with disseminated disease $[2,6,12,13]$.

Distant metastases occur in $10-20 \%$ of all patients at presentation, and the most common sites include the ovaries, peritoneum, and liver. Kanthan et al. [14] reported a histologically proven case of a GCC metastasizing to the lung in a 60-year-old male. However, in that case, as well as in all cases of metastatic GCCs in our previously reported series [6], the Ki67 proliferation index was $>20 \%$ and the tumours, according to the new GCC classification, were 'adenocarcinomas ex-GCC' of either 'signet ring cell type' or 'poorly differentiated adenocarcinoma type'. In our case, the Ki67 proliferation index was surprisingly low $(<5 \%)$, and therefore this is the first case in the literature with a lung metastasis from a typical GCC.

The normal levels of chromogranin A and the absence of uptake in Octreoscan in our patient, despite advanced disease, confirm our previous findings about the limited values of Octreoscan in GCC in contrast to other metastatic appendiceal or midgut carcinoids.

In GCCs, according to our previous observation [6], 18FDG-PET seems to be a very good imaging modality for assessment of disease extent. In our patient, it showed uptake in the right hilar mass. The absence of uptake in the abdominal lymph nodes may have been due to their small size. ${ }^{18}$ FDG-PET, in general, seems to be more sensitive than Octreoscan in NET and high-grade tumour patients. In our case, the fact that 18FDG-PET showed uptake in the lung lesion, despite the absence of a high proliferation index, probably reflects the aggressiveness of GCCs.

In patients with advanced GCC, systemic chemotherapy is considered, although there is no well-established chemotherapy regimen for these patients. Most centres use the FOLFOX regimen as treatment protocol for colon cancer. Unfortunately, our patient did not respond to FOLFOX chemotherapy.

This case demonstrates the supra-diaphragmatic metastatic potential of GCCs even in those patients with a low-grade histology. 
The overall 5-year survival for GCC ranges between $40-75 \%$ and depends on the tumour stage at presentation. ENETS guidelines recommend lifelong screening for synchronous or metachronous malignancies. A comparable follow-up plan to colorectal adenocarcinomas is advised [8].

On the basis of these findings, we would recommend consideration of a contrast CT of the chest, abdomen, and pelvis for all patients with a GCC tumour regardless of histological grade because of their potential to metastasize to distant sites, including the lungs. In case of indeterminate findings, FDG-PET seems to be helpful and, if still in doubt, a guided biopsy of the suspicious lesion may be required to confirm the diagnosis.
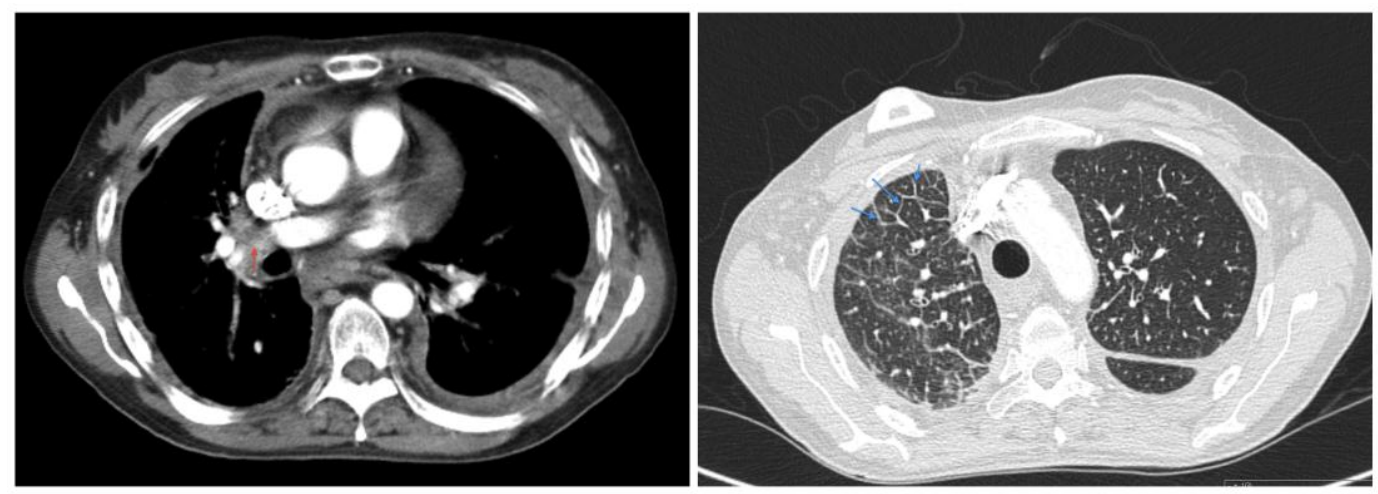

Fig. 1. Axial slice of the contrast-enhanced CT showing the right hilar mass (red arrow) and the rightsided interlobular septal thickening (blue arrow). 


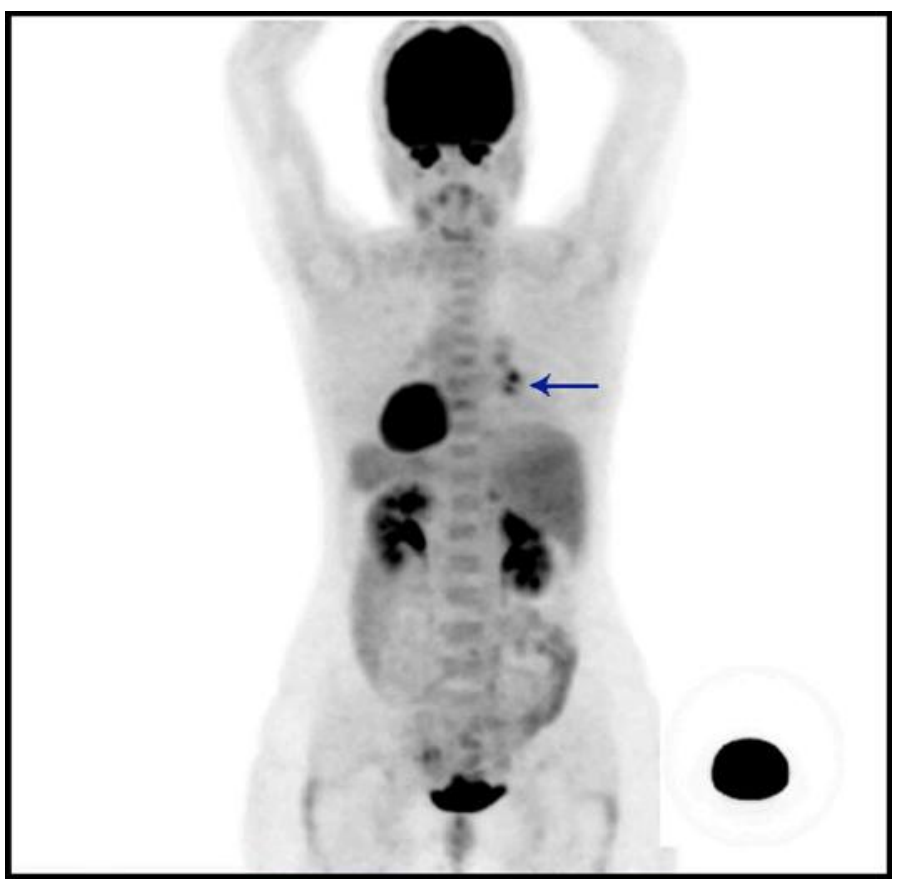

Fig. 2. The hilar lesion (arrow) demonstrates low-to-moderate avidity on ${ }^{18 F D G ~ P E T ~ a n d ~ t h e r e ~ i s ~ n o ~}$ FDG uptake elsewhere. 

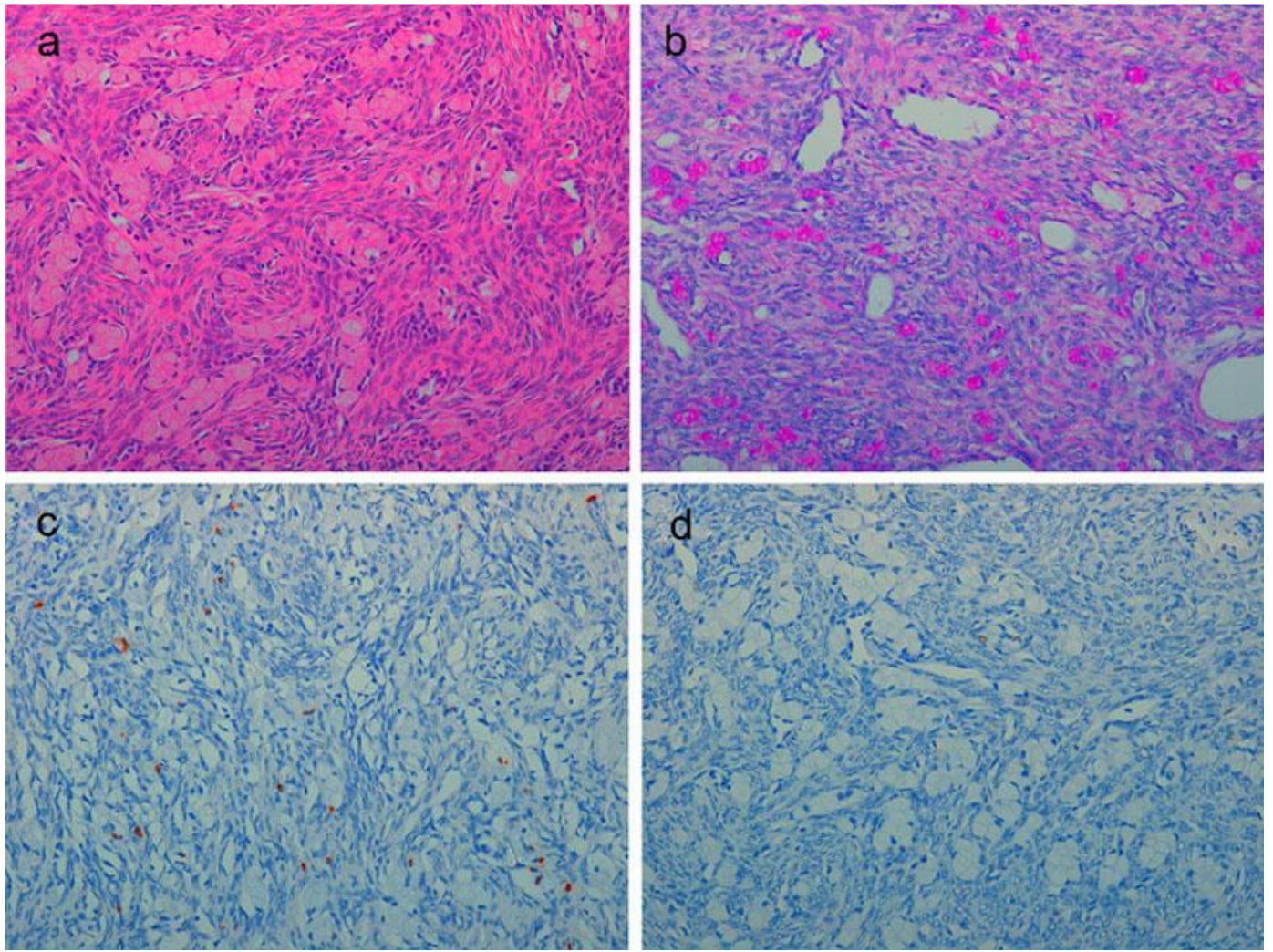

Fig. 3. a HE-stained section $(\times 20)$ showing ovarian stroma infiltrated by a GCC composed of small, rounded nests of signet-ring-like cells resembling normal intestinal goblet cells and scattered eosinophilic neuroendocrine cells (b). ABDPAS ( $\times 20)$ : mucin staining is intensively positive within goblet cells (c). Chromogranin A ( $\times 20)$ : immunohistochemistry for chromogranin A highlights the scattered endocrine cell component. The goblet cells are negative (d). Ki67 ( $\times 20)$ : the Ki67 proliferation index is low $(<2 \%)$.

\section{References}

1 Goede AC, Caplin ME, Winslet MC: Carcinoid tumour of the appendix. Br J Surg 2003;90:1317-1322.

-2 Pahlavan PS, Kanthan R: Goblet cell carcinoid of the appendix. World J Surg Oncol 2005;3:36.

-3 Subbuswamy SG, Gibbs NM, Ross CF, Morson BC: Goblet cell carcinoid of the appendix. Cancer 1974;34:338-344.

4 Hemminki K, Li X: Incidence trends and risk factors of carcinoid tumors: a nationwide epidemiologic study from Sweden. Cancer 2001;92:2204-2210.

-5 Plockinger U, Couvelard A, Falconi M, Sundin A, Salazar R, Christ E, de Herder WW, Gross D, Knapp WH, Knigge UP, Kulke MH, Pape UF, Frascati Consensus Conference participants: Consensus guidelines for the management of patients with digestive neuroendocrine tumours: well-differentiated tumour/carcinoma of the appendix and goblet cell carcinoma. Neuroendocrinology 2008;87:20-30.

-6 Toumpanakis C, Standish RA, Baishnab E, Winslet MC, Chaplin ME: Goblet cell carcinoid tumors (adenocarcinoid) of the appendix. Dis Colon Rectum 2007;50:315-322.

-7 Tang LH, Shia J, Soslow RA, Dhall D, Wong WD, O’Reilly E, Qin J, Paty P, Weiser MR, Guillem J, Temple L, Sobin LH, Klimstra DS: Pathologic classification and clinical behavior of the spectrum of goblet cell carcinoid tumors of the appendix. Am J Surg Pathol 2008;32:1429-1443.

8 Pape UF, Perren A, Niederle B, Gross D, Gress T, Costa F, Arnold R, Denecke T, Plöckinger U, Salazar R, Grossman A, Barcelona Consensus Conference participants: ENETS Consensus Guidelines for the 
management of patients with neuroendocrine neoplasms from the jejuno-ileum and the appendix including goblet cell carcinomas. Neuroendocrinology 2012;95:135-156.

-9 Zirkin RM, Brown S, Hertz M: Adenocarcinoid of appendix presenting as bilateral ovarian tumors. A case report with histochemical and ultrastructural studies. Diagn Gynecol Obstet 1980;2:269-274.

10 Argani P, van Hoeven KH, Artymyshyn RL: Diagnosis of metastatic appendiceal adenocarcinoid in liver by fine-needle aspiration cytology. Diagn Cytopathol 1995;12:59-61.

11 Zafar S, Chen H, Sun W, Das K: Cytology of metastatic appendiceal goblet cell carcinoid in pleural effusion fluid: a case report. Diagn Cytopathol 2008;36:894-898.

12 Mahteme H, Sugarbaker PH: Treatment of peritoneal carcinomatosis from adenocarcinoid of appendiceal origin. Br J Surg 2004;91:1168-1173.

13 Garin L, Corbinais S, Boucher E, Blanchot J, Le Guilcher P, Raoul JL: Adenocarcinoid of the appendix vermiformis: complete and persistent remission after chemotherapy (folfox) of a metastatic case. Dig Dis Sci 2002;47:2760-2762.

14 Kanthan R, Saxena A, Kanthan SC: Goblet cell carcinoids of the appendix: immunophenotype and ultrastructural study. Arch Pathol Lab Med 2001;125:386-390. 